\title{
POSITIVE SOLUTIONS FOR A CLASS OF $p(x)$-LAPLACIAN PROBLEMS
}

\author{
G. A. AFROUZI and H. GHORBANI \\ Department of Mathematics, Faculty of Basic Sciences, \\ Mazandaran University, Babolsar, Iran \\ e-mail:afrouzi@umz.ac.ir
}

(Received 18 September 2008; accepted 8 January 2009)

Abstract. We consider the system

$$
\begin{cases}-\Delta_{p(x)} u=\lambda_{1} f(v)+\mu_{1} h(u) & \text { in } \Omega \\ -\Delta_{q(x)} v=\lambda_{2} g(u)+\mu_{2} \gamma(v) & \text { in } \Omega \\ u=v=0 & \text { on } \partial \Omega\end{cases}
$$

where $p(x), q(x) \in C^{1}\left(R^{N}\right)$ are radial symmetric functions such that $\sup |\nabla p(x)|<$ $\infty, \sup |\nabla q(x)|<\infty$ and $1<\inf p(x) \leq \sup p(x)<\infty, 1<\inf q(x) \leq \sup q(x)<\infty$, where $-\Delta_{p(x)} u=-\operatorname{div}\left(|\nabla u|^{p(x)-2} \nabla u\right),-\Delta_{q(x)} v=-\operatorname{div}\left(|\nabla v|^{q(x)-2} \nabla v\right)$, respectively are called $p(x)$-Laplacian and $q(x)$-Laplacian, $\lambda_{1}, \lambda_{2}, \mu_{1}$ and $\mu_{2}$ are positive parameters and $\Omega=B(0, R) \subset R^{N}$ is a bounded radial symmetric domain, where $R$ is sufficiently large. We prove the existence of a positive solution when

$$
\lim _{u \rightarrow+\infty} \frac{f\left(M(g(u))^{\frac{1}{q^{-}-1}}\right)}{u^{p^{-}-1}}=0,
$$

for every $M>0, \lim _{u \rightarrow+\infty} \frac{h(u)}{u^{p^{-}-1}}=0$ and $\lim _{u \rightarrow+\infty} \frac{\gamma(u)}{u^{q^{-}-1}}=0$. In particular, we do not assume any sign conditions on $f(0), g(0), h(0)$ or $\gamma(0)$.

AMS Subject Classification. 35J60, 35B30, 35B40.

1. Introduction. The study of differential equations and variational problems with non-standard $p(x)$-growth conditions has been a new and interesting topic. Many results have been obtained on this kind of problem, for example, $[3-8,10,11,13]$. In $[6,7]$ Fan and Zhao give the regularity of weak solutions for differential equations with non-standard $p(x)$-growth conditions. Zhang in [12] investigated the existence of positive solutions of the system

$$
\begin{cases}-\Delta_{p(x)} u=f(v) & \text { in } \Omega \\ -\Delta_{q(x)} v=g(u) & \text { in } \Omega, \\ u=v=0 & \text { on } \partial \Omega\end{cases}
$$


where $p(x) \in C^{1}\left(R^{N}\right)$ is a function and $\Omega \subset R^{N}$ is a bounded domain. The operator $-\Delta_{p(x)} u=-\operatorname{div}\left(|\nabla u|^{p(x)-2} \nabla u\right)$ is called $p(x)$-Laplacian. Especially, if $p(x) \equiv p$ (a constant), (1) is the well-known $p$-Laplacian systems. There are many papers on the existence of solutions for $p$-Laplacian elliptic systems, for example, [1-9].

In [9] the authors consider the existence of positive weak solutions for the following $p$-Laplacian problems:

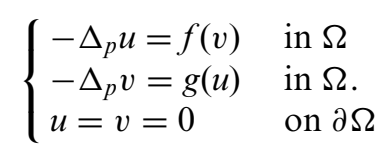

The first eigenfunction is used for constructing the subsolution of $p$-Laplacian problems successfully. On the condition of

$$
\lim _{u \rightarrow+\infty} \frac{f\left(M(g(u))^{\frac{1}{p-1}}\right)}{u^{p-1}}=0, \quad \forall M>0,
$$

the authors show the existence of positive solutions for problem (2).

In this paper, we mainly consider the existence of positive solutions of the system

$$
\begin{cases}-\Delta_{p(x)} u=\lambda_{1} f(v)+\mu_{1} h(u) & \text { in } \Omega \\ -\Delta_{q(x)} v=\lambda_{2} g(u)+\mu_{2} \gamma(v) & \text { in } \Omega \\ u=v=0 & \text { on } \partial \Omega\end{cases}
$$

where $p(x), q(x) \in C^{1}\left(R^{N}\right)$ are functions, $\lambda_{1}, \lambda_{2}, \mu_{1}$ and $\mu_{2}$ are positive parameters and $\Omega \subset R^{N}$ is a bounded domain.

In order to deal with $p(x)$-Laplacian problems, we need some theories on spaces $L^{p(x)}(\Omega)$, and $W^{1, p(x)}(\Omega)$ and properties of $p(x)$-Laplacian which we will use later (see [8]). If $\Omega \subset R^{N}$ is an open domain, then

$$
\begin{aligned}
& C_{+}(\Omega)=\{h \mid h \in C(\Omega), h(x)>1 \text { for } x \in \Omega\}, \\
& h^{+}=\sup _{x \in \Omega} h(x), \quad h^{-}=\inf _{x \in \Omega} h(x), \quad \text { for any } h \in C(\Omega), \\
& L^{p(x)}(\Omega)=\left\{u \mid u \text { is a measurable real-valued function, } \int_{\Omega}|u|^{p(x)} d x<\infty\right\}
\end{aligned}
$$

Throughout the paper, we will assume that $p, q \in C_{+}(\Omega)$ and

$$
\begin{aligned}
& 1<\inf _{x \in R^{N}} p(x) \leq \sup _{x \in R^{N}} p(x)<N, \\
& 1<\inf _{x \in R^{N}} q(x) \leq \sup _{x \in R^{N}} q(x)<N .
\end{aligned}
$$

We can introduce the norm on $L^{p(x)}(\Omega)$ by

$$
|u|_{p(x)}=\inf \left\{\lambda>\left.0\left|\int_{\Omega}\right| \frac{u(x)}{\lambda}\right|^{p(x)} d x \leq 1\right\},
$$

and $\left(L^{p(x)}(\Omega),|\cdot|_{p(x)}\right)$ becomes a Banach space, which we call generalised Lebesgue space.

The space $\left(L^{p(x)}(\Omega),|\cdot|_{p(x)}\right)$ is a separable, reflexive and uniformly convex Banach space (see [8, Theorem 1.10, 1.14]).

The space $W^{1, p(x)}(\Omega)$ is defined by

$$
W^{1, p(x)}(\Omega)=\left\{u \in L^{p(x)}(\Omega) \| \nabla u \mid \in L^{p(x)}(\Omega)\right\},
$$


and it can be equipped with the norm

$$
\|u\|=|u|_{p(x)}+|\nabla u|_{p(x)}, \quad \forall u \in W^{1, p(x)}(\Omega) .
$$

We denote by $W_{0}^{1, p(x)}(\Omega)$ the closure of $C_{0}^{\infty}(\Omega)$ in $W^{1, p(x)}(\Omega) . W^{1, p(x)}(\Omega)$ and $W_{0}^{1, p(x)}(\Omega)$ are separable, reflexive and uniformly convex Banach spaces (see [8, Theorem 2.1]). We define that if

$$
(L(u), v)=\int_{R^{N}}|\nabla u|^{p(x)-2} \nabla u \nabla v d x, \quad \forall u, v \in W^{1, p(x)}(\Omega),
$$

then $L: W^{1, p(x)}(\Omega) \rightarrow\left(W^{1, p(x)}(\Omega)\right)^{*}$ is a continuous, bounded and strictly monotone operator and is also a homeomorphism (see [4, Theorem 3.11]). If $u, v \in$ $W_{0}^{1, p(x)}(\Omega),(u, v)$ is called a weak solution of (3) which satisfies

$$
\begin{array}{ll}
\int_{\Omega}|\nabla u|^{p(x)-2} \nabla u \nabla \xi d x=\int_{\Omega}\left(\lambda_{1} f(v)+\mu_{1} h(u)\right) \xi d x, & \forall \xi \in W_{0}^{1, p(x)}(\Omega), \\
\int_{\Omega}|\nabla v|^{q(x)-2} \nabla v \nabla \xi d x=\int_{\Omega}\left(\lambda_{2} g(u)+\mu_{2} \gamma(v)\right) \xi d x, & \forall \xi \in W_{0}^{1, p(x)}(\Omega) .
\end{array}
$$

We make the following assumptions

(H.1) $p(x), q(x) \in C^{1}\left(R^{N}\right)$ are radial symmetric and $\sup |\nabla p(x)|<\infty$, sup $\mid$ $\nabla q(x) \mid<\infty$.

(H.2) $\Omega=B(0, R)=\{x|| x \mid<R\}$ is a ball, where $R>0$ is a sufficiently large constant.

(H.3) $f, g, h, \gamma:[0, \infty) \rightarrow R$ are $C^{1}$, monotone functions such that

$$
\lim _{u \rightarrow+\infty} f(u)=\lim _{u \rightarrow+\infty} g(u)=\lim _{u \rightarrow+\infty} h(u)=\lim _{u \rightarrow+\infty} \gamma(u)=+\infty .
$$

(H.4) $\lim _{u \rightarrow+\infty} \frac{f\left(M(g(u))^{\frac{1}{q^{-}-1}}\right)}{u^{p^{-}-1}}=0$, for every $M>0$.

(H.5) $\lim _{u \rightarrow+\infty} \frac{h(u)}{u^{p^{-}-1}}=\lim _{u \rightarrow+\infty} \frac{\gamma(u)}{u^{q^{-}-1}}=0$.

We shall establish the following theorem.

\section{Main results.}

THEOREM 1. If (H.1)-(H.5) hold, then (3) has a positive solution.

Proof. We shall establish Theorem 1 by constructing a positive subsolution $\left(\phi_{1}, \phi_{2}\right)$ and supersolution $\left(z_{1}, z_{2}\right)$ of (3), such that $\phi_{1} \leq z_{1}$ and $\phi_{2} \leq z_{2}$. That is, $\left(\phi_{1}, \phi_{2}\right)$ and $\left(z_{1}, z_{2}\right)$ satisfy

$$
\begin{aligned}
& \int_{\Omega}\left|\nabla \phi_{1}\right|^{p(x)-2} \nabla \phi_{1} \cdot \nabla \xi d x \leq \lambda_{1} \int_{\Omega} f\left(\phi_{2}\right) \xi d x+\mu_{1} \int_{\Omega} h\left(\phi_{1}\right) \xi d x, \\
& \int_{\Omega}\left|\nabla \phi_{2}\right|^{q(x)-2} \nabla \phi_{1} \cdot \nabla \xi d x \leq \lambda_{2} \int_{\Omega} g\left(\phi_{1}\right) \xi d x+\mu_{2} \int_{\Omega} \gamma\left(\phi_{2}\right) \xi d x,
\end{aligned}
$$

and

$$
\begin{aligned}
& \int_{\Omega}\left|\nabla \phi_{1}\right|^{p(x)-2} \nabla \phi_{1} \cdot \nabla \xi d x \geq \lambda_{1} \int_{\Omega} f\left(\phi_{2}\right) \xi d x+\mu_{1} \int_{\Omega} h\left(\phi_{1}\right) \xi d x, \\
& \int_{\Omega}\left|\nabla \phi_{2}\right|^{q(x)-2} \nabla \phi_{1} \cdot \nabla \xi d x \geq \lambda_{2} \int_{\Omega} g\left(\phi_{1}\right) \xi d x+\mu_{2} \int_{\Omega} \gamma\left(\phi_{2}\right) \xi d x,
\end{aligned}
$$

for all $\xi \in W_{0}^{1, p(x)}(\Omega)$ with $\xi \geq 0$. Then (3) has a positive solution. 
Step 1. We construct a subsolution of (3).

Denote

$$
\begin{array}{ll}
a_{1}=\frac{\inf p(x)-1}{4(\sup |\nabla p(x)|+1)}, & R_{1}=\frac{R-a_{1}}{2}, \\
a_{2}=\frac{\inf q(x)-1}{4(\sup |\nabla q(x)|+1)}, & R_{2}=\frac{R-a_{2}}{2},
\end{array}
$$

and let $k_{0}>0$ such that $f(u), g(u), h(u), \gamma(u) \geq-k_{0}$ for all $u \geq 0$, and let

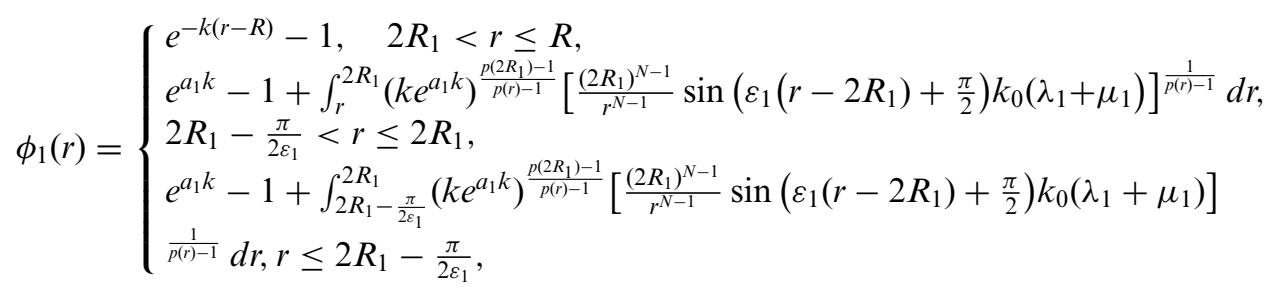

where $R_{1}$ is sufficiently large and $\varepsilon_{1}$ is a small positive constant which satisfies

$$
R_{1} \leq 2 R_{1}-\frac{\pi}{2 \varepsilon_{1}}
$$

and let

$$
\phi_{2}(r)=\left\{\begin{array}{l}
e^{-k(r-R)}-1, \quad 2 R_{2}<r \leq R, \\
e^{a_{2} k}-1+\int_{r}^{2 R_{2}}\left(k e^{a_{2} k}\right)^{\frac{q\left(2 R_{2}\right)-1}{q(r)-1}}\left[\frac{\left(2 R_{2}\right)^{N-1}}{r^{N-1}} \sin \left(\varepsilon_{2}\left(r-2 R_{2}\right)+\frac{\pi}{2}\right) k_{0}\left(\lambda_{2}+\mu_{2}\right)\right]^{\frac{1}{q(r)-1}} d r, \\
2 R_{2}-\frac{\pi}{2 \varepsilon_{2}}<r \leq 2 R_{2}, \\
e^{a_{2} k}-1+\int_{2 R_{2}-\frac{\pi}{2 \varepsilon_{2}}}^{2 R_{2}}\left(k e^{a_{2} k}\right)^{\frac{q\left(2 R_{2}\right)-1}{q(r)-1}}\left[\frac{\left(2 R_{2}\right)^{N-1}}{r^{N-1}} \sin \left(\varepsilon_{2}\left(r-2 R_{2}\right)+\frac{\pi}{2}\right) k_{0}\left(\lambda_{2}+\mu_{2}\right)\right] \\
\frac{1}{q(r)-1} d r, r \leq 2 R_{2}-\frac{\pi}{2 \varepsilon_{2}},
\end{array}\right.
$$

where $R_{2}$ is sufficiently large and $\varepsilon_{2}$ is a small positive constant which satisfies

$$
R_{2} \leq 2 R_{2}-\frac{\pi}{2 \varepsilon_{2}}
$$
Since

In the following, we will prove that $\left(\phi_{1}, \phi_{2}\right)$ is a subsolution of (3).

$$
\phi_{1}^{\prime}(r)=\left\{\begin{array}{l}
e^{-k(r-R)}-1, \quad 2 R_{1}<r \leq R, \\
-\left(k e^{a_{1} k}\right)^{\frac{p\left(2 R_{1}\right)-1}{p(r)-1}}\left[\frac{\left(2 R_{1}\right)^{N-1}}{r^{N-1}} \sin \left(\varepsilon_{1}\left(r-2 R_{1}\right)+\frac{\pi}{2}\right) k_{0}\left(\lambda_{1}+\mu_{1}\right)\right]^{\frac{1}{p(r)-1}} d r, \\
2 R_{1}-\frac{\pi}{2 \varepsilon_{1}}<r \leq 2 R_{1}, \\
0, \quad 0 \leq r \leq 2 R_{1}-\frac{\pi}{2 \varepsilon_{1}},
\end{array}\right.
$$

and

$$
\phi_{2}^{\prime}(r)=\left\{\begin{array}{l}
e^{-k(r-R)}-1, \quad 2 R_{2}<r \leq R, \\
-\left(k e^{a_{2} k}\right)^{\frac{q\left(2 R_{2}\right)-1}{q(r)-1}}\left[\frac{\left(2 R_{2}\right)^{N-1}}{r^{N-1}} \sin \left(\varepsilon_{2}\left(r-2 R_{2}\right)+\frac{\pi}{2}\right) k_{0}\left(\lambda_{2}+\mu_{2}\right)\right]^{\frac{1}{q(r)-1}} d r, \\
2 R_{2}-\frac{\pi}{2 \varepsilon_{2}}<r \leq 2 R_{2}, \\
0, \quad 0 \leq r \leq 2 R_{2}-\frac{\pi}{2 \varepsilon_{2}}
\end{array}\right.
$$


it is easy to see that $\phi_{1}, \phi_{2} \geq 0$ are decreasing and $\phi_{1}, \phi_{2} \in C^{1}([0, R]), \phi_{1}(x)=\phi_{1}(|x|) \in$ $C^{1}(\bar{\Omega})$ and $\phi_{2}(x)=\phi_{2}(|x|) \in C^{1}(\bar{\Omega})$.

Let $r=|x|$. By computation,

$$
-\Delta_{p(x)} \phi_{1}=-\operatorname{div}\left(\left|\nabla \phi_{1}(x)\right|^{p(x)-2} \nabla \phi_{1}(x)\right)=-\left(r^{N-1}\left|\phi_{1}^{\prime}(r)\right|^{p(r)-2} \phi_{1}^{\prime}(r)\right)^{\prime} / r^{N-1},
$$

so then

$$
-\Delta_{p(x)} \phi_{1}=\left\{\begin{array}{l}
\left(k e^{-k(r-R)}\right)^{p(r)-1}\left[-k(p(r)-1)+p^{\prime}(r) \ln k-k p^{\prime}(r)(r-R)+\frac{N-1}{r}\right], \\
2 R_{1}<r \leq R, \\
\varepsilon_{1}\left(\frac{2 R_{1}}{r}\right)^{N-1}\left(k e^{a_{1} k}\right)^{p\left(2 R_{1}\right)-1} \cos \left(\varepsilon_{1}\left(r-2 R_{1}\right)+\frac{\pi}{2}\right)\left(\lambda_{1}+\mu_{1}\right), \\
2 R_{1}-\frac{\pi}{2 \varepsilon_{1}}<r \leq 2 R_{1}, 0, \quad 0 \leq r \leq 2 R_{1}-\frac{\pi}{2 \varepsilon_{1}} .
\end{array}\right.
$$

If $k$ is sufficiently large, when $2 R_{1}<r \leq R$, then we have

$$
-\Delta_{p(x)} \phi_{1} \leq-k\left[\inf p(x)-1-\sup |\nabla p(x)|\left(\frac{\ln k}{k}+R-r\right)+\frac{N-1}{k r}\right] \leq-k a_{1} .
$$

As $a_{1}$ is a constant dependent only on $p(x)$, if $k$ is big enough, such that

$$
-k a_{1}<-\left(\lambda_{1}+\mu_{1}\right) k_{0}
$$

then we have

$$
-\Delta_{p(x)} \phi_{1} \leq-\left(\lambda_{1}+\mu_{1}\right) k_{0} \leq \lambda_{1} f\left(\phi_{2}\right)+\mu_{1} h\left(\phi_{1}\right), \quad 2 R_{1}<|x| \leq R .
$$

If $k$ is sufficiently large, then

$$
f\left(e^{a_{2} k}-1\right) \geq 1, h\left(e^{a_{1} k}-1\right) \geq 1, g\left(e^{a_{1} k}-1\right) \geq 1, \gamma\left(e^{a_{2} k}-1\right) \geq 1,
$$

where $k$ is dependent on $f, h, g, \gamma$ and $p, q$ and independent on $R$. Since

$$
\begin{aligned}
-\Delta_{p(x)} \phi_{1} & =\varepsilon_{1}\left(\frac{2 R_{1}}{r}\right)^{N-1}\left(k e^{a_{1} k}\right)^{p\left(2 R_{1}\right)-1} \cos \left(\varepsilon_{1}\left(r-2 R_{1}\right)+\frac{\pi}{2}\right)\left(\lambda_{1}+\mu_{1}\right) \\
& \leq \varepsilon_{1}\left(\lambda_{1}+\mu_{1}\right) 2^{N} k^{p^{+}} e^{a_{1} k p^{+}}, \quad 2 R_{1}-\frac{\pi}{2 \varepsilon_{1}}<|x| \leq 2 R_{1},
\end{aligned}
$$

let

$$
\varepsilon_{1}=2^{-N} k^{-p^{+}} e^{-a_{1} k p^{+}}
$$

Then we have

$$
-\Delta_{p(x)} \phi_{1} \leq \lambda_{1}+\mu_{1} \leq \lambda_{1} f\left(\phi_{2}\right)+\mu_{1} h\left(\phi_{1}\right), \quad 2 R_{1}-\frac{\pi}{2 \varepsilon_{1}}<|x| \leq 2 R_{1} .
$$

Obviously,

$$
-\Delta_{p(x)} \phi_{1}=0 \leq \lambda_{1}+\mu_{1} \leq \lambda_{1} f\left(\phi_{2}\right)+\mu_{1} h\left(\phi_{1}\right), \quad|x| \leq 2 R_{1}-\frac{\pi}{2 \varepsilon_{1}} .
$$

Since $\phi_{1}(x) \in C^{1}(\Omega)$, combining (4), (5) and (6), we have

$$
-\Delta_{p(x)} \phi_{1} \leq \lambda_{1} f\left(\phi_{2}\right)+\mu_{1} h\left(\phi_{1}\right), \quad \text { for } \quad \text { a.e. } x \in \Omega \text {. }
$$


Similarly we have

$$
-\Delta_{q(x)} \phi_{2} \leq \lambda_{2} g\left(\phi_{1}\right)+\mu_{2} \gamma\left(\phi_{2}\right), \quad \text { for } \quad \text { a.e. } x \in \Omega .
$$

Since $\phi_{1}(x), \phi_{2}(x) \in C^{1}(\bar{\Omega})$, it is easy to see that $\left(\phi_{1}, \phi_{2}\right)$ is a subsolution of (3).

Step 2. We construct a supersolution of (3).

Let $z_{1}$ be a radial solution of

$$
-\Delta_{p(x)} z_{1}(x)=\left(\lambda_{1}+\mu_{1}\right) \mu, \quad \text { in } \quad \Omega, \quad z_{1}=0 \quad \text { on } \quad \partial \Omega .
$$

We denote that if $z_{1}=z_{1}(r)=z_{1}(|x|)$, then $z_{1}$ satisfies

$$
-\left(r^{N-1}\left|z_{1}^{\prime}\right|^{p(r)-2} z_{1}^{\prime}\right)^{\prime}=r^{N-1}\left(\lambda_{1}+\mu_{1}\right) \mu, \quad z_{1}(R)=0, z_{1}^{\prime}(0)=0,
$$

and so

$$
z_{1}^{\prime}=-\left|\frac{r\left(\lambda_{1}+\mu_{1}\right) \mu}{N}\right|^{\frac{1}{p(r)-1}}
$$

and

$$
z_{1}=\int_{r}^{R}\left|\frac{r\left(\lambda_{1}+\mu_{1}\right) \mu}{N}\right|^{\frac{1}{p(r)-1}} d r
$$

We denote that if $\beta=\beta\left(\left(\lambda_{1}+\mu_{1}\right) \mu\right)=\max _{0 \leq r \leq R} z_{1}(r)$, then

$$
\beta\left(\left(\lambda_{1}+\mu_{1}\right) \mu\right)=\int_{0}^{R}\left|\frac{r\left(\lambda_{1}+\mu_{1}\right) \mu}{N}\right|^{\frac{1}{p(r)-1}} d r=\left(\left(\lambda_{1}+\mu_{1}\right) \mu\right)^{\frac{1}{p(t)-1}} \int_{0}^{R}\left|\frac{r}{N}\right|^{\frac{1}{p(r)-1}} d r
$$

where $t \in[0,1]$. Since $\int_{0}^{R}\left|\frac{r}{N}\right|^{\frac{1}{p(r)-1}} d r$ is a constant, then there exists a positive constant $C \geq 1$ such that

$$
\frac{1}{C}\left(\left(\lambda_{1}+\mu_{1}\right) \mu\right)^{\frac{1}{p^{+}-1}} \leq \beta\left(\left(\lambda_{1}+\mu_{1}\right) \mu\right)=\max _{0 \leq r \leq R} z_{1}(r) \leq C\left(\left(\lambda_{1}+\mu_{1}\right) \mu\right)^{\frac{1}{p^{-}-1}} .
$$

We consider

$$
\begin{cases}-\Delta_{p(x)} z_{1}=\left(\lambda_{1}+\mu_{1}\right) \mu & \text { in } \Omega \\ -\Delta_{q(x)} z_{2}=\left(\lambda_{2}+\mu_{2}\right) g\left(\beta\left(\left(\lambda_{1}+\mu_{1}\right) \mu\right)\right) & \text { in } \Omega \\ z_{1}=z_{2}=0 & \text { on } \partial \Omega\end{cases}
$$

and then we shall prove that $\left(z_{1}, z_{2}\right)$ is a supersolution for (3).

For $\xi \in W^{1, p(x)}(\Omega)$ with $\xi \geq 0$ it is easy to see that

$$
\begin{aligned}
\int_{\Omega}\left|\nabla z_{2}\right|^{q(x)-2} \nabla z_{2} \cdot \nabla \xi d x & =\int_{\Omega}\left(\lambda_{2}+\mu_{2}\right) g\left(\beta\left(\left(\lambda_{1}+\mu_{1}\right) \mu\right)\right) \xi d x \\
& \geq \int_{\Omega} \lambda_{2} g\left(z_{1}\right) \xi d x+\int_{\Omega} \mu_{2} g\left(\beta\left(\left(\lambda_{1}+\mu_{1}\right) \mu\right)\right) \xi d x .
\end{aligned}
$$

By (H.5) for $\mu$ large enough, we have

$$
g\left(\beta\left(\left(\lambda_{1}+\mu_{1}\right) \mu\right)\right) \geq \gamma\left(\left[\left(\lambda_{2}+\mu_{2}\right)\left(g\left(\beta\left(\left(\lambda_{1}+\mu_{1}\right) \mu\right)\right)\right)\right]^{\frac{1}{q^{-}-1}}\right) \geq \gamma\left(z_{2}\right) .
$$


Hence

$$
\int_{\Omega}\left|\nabla z_{2}\right|^{q(x)-2} \nabla z_{2} \cdot \nabla \xi d x \geq \int_{\Omega} \lambda_{2} g\left(z_{1}\right) \xi d x+\int_{\Omega} \mu_{2} \gamma\left(z_{2}\right) \xi d x
$$

Also

$$
\int_{\Omega}\left|\nabla z_{1}\right|^{p(x)-2} \nabla z_{1} \cdot \nabla \xi d x=\int_{\Omega}\left(\lambda_{1}+\mu_{1}\right) \mu \xi d x .
$$

Similar to (8), we have

$$
\max _{0 \leq r \leq R} z_{2}(r) \leq C\left[\left(\lambda_{2}+\mu_{2}\right) g\left(\beta\left(\left(\lambda_{1}+\mu_{1}\right) \mu\right)\right)\right]^{\frac{1}{\left(q^{-}-1\right)}}
$$

By (H.4) and (H.5), when $\mu$ is sufficiently large, according to (8), we have

$$
\begin{aligned}
\left(\lambda_{1}+\mu_{1}\right) \mu & \geq\left[\frac{1}{C} \beta\left(\left(\lambda_{1}+\mu_{1}\right) \mu\right)\right]^{p^{-}-1} \\
& \geq \lambda_{1} f\left[C\left(\left[\left(\lambda_{2}+\mu_{2}\right)\left(g\left(\beta\left(\left(\lambda_{1}+\mu_{1}\right) \mu\right)\right)\right)\right]^{\frac{1}{\left.q^{-}-1\right)}}\right]+\mu_{1} h\left(\beta\left(\left(\lambda_{1}+\mu_{1}\right) \mu\right)\right.\right. \\
& \geq \lambda_{1} f\left(z_{2}\right)+\mu_{1} h\left(z_{1}\right),
\end{aligned}
$$

and so

$$
\int_{\Omega}\left|\nabla z_{1}\right|^{p(x)-2} \nabla z_{1} \cdot \nabla \xi d x \geq \int_{\Omega} \lambda_{1} f\left(z_{2}\right) \xi d x+\int_{\Omega} \mu_{1} h\left(z_{1}\right) \xi d x
$$

According to (9) and (10), we can conclude that $\left(z_{1}, z_{2}\right)$ is a supersolution of (3).

Let $\mu$ be sufficiently large; then from (7) and the definition of $\left(\phi_{1}, \phi_{2}\right)$, it is easy to see that $\phi_{1} \leq z_{1}$ and $\phi_{2} \leq z_{2}$. This completes the proof.

\section{REFERENCES}

1. J. Ali and R. Shivaji, Positive solutions for a class of $p$-Laplacian systems with multiple parameters, J. Math. Anal. Appl. 335 (2007), 1013-1019.

2. C. H. Chen, On positive weak solutions for a class of quasilinear elliptic systems, Nonlinear Anal. 62 (2005), 751-756.

3. X. L. Fan, H. Q. Wu, and F. Z. Waang, Hartman-type results for $p(t)$-Laplacian systems, Nonlinear Anal. 52 (2003), 585-594.

4. X. L. Fan and Q. H. Zhang, Existence of solutions for $p(x)$-Laplacian Dirichlet problem, Nonlinear Anal. 52 (2003), 1843-1852.

5. X. L. Fan, Q. H. Zhang and D. Zhao Eigenvalues of $p(x)$-Laplacian Dirichlet problem, J. Math. Anal. Appl. 302 (2005), 306-317.

6. X. L. Fan and D. Zhao, A class of De Giorgi type and Hölder continuity, Nonlinear Anal. TMA 36 (1999), 295-318.

7. X. L. Fan and D. Zhao, The quasi-minimizer of integral functionals with $m(x)$ growth conditions, Nonlinear Anal. TMA 39 (2000), 807-816.

8. X. L. Fan and D. Zhao, On the spaces $L^{p(x)}(\Omega)$ and $W^{m, p(x)}(\Omega)$, J. Math. Anal. Appl. 263 (2001), 424- 446.

9. D. D. Hai and R. Shivaji, An existence result on positive solutions for a class of p-Laplacian systems, Nonlinear Anal. 56 (2004), 1007-1010. 
10. M. Rûzicka, Electrorheological fluids: Modeling and mathematical theory, Lecture Notes in Math, vol. 1784 (Springer-Verlag, Berlin, 2000).

11. Q. H. Zhang, Existence of positive solutions for a class of $p(x)$-Laplacian systems, J. Math. Anal. Appl. 302 (2005), 306-317.

12. Q. H. Zhang, Existence of positive solutions for elliptic systems with nonstandard $p(x)$-growth conditions via sub-supersolution method, Nonlinear Anal. 67 (2007), 1055-1067.

13. V. V. Zhikov, Averaging of functionals of the calculus of variations and elasticity theory, Math. USSR Izv. 29 (1987), 33-36. 